\title{
Health promotion 'on steroids': the value of an experiential approach to promote rapid HIV testing in NSW, Australia
}

\author{
a NSW Public Health Training Program, NSW Ministry of Health, Sydney, Australia \\ ${ }^{\mathrm{b}}$ Centre for Population Health, NSW Ministry of Health, Sydney, Australia \\ c Corresponding author: nrobe@doh.health.nsw.gov.au
}

Nick Roberts ${ }^{a, c}$, Jo Holden ${ }^{\text {, Timothy Duck }}$ and Samara Kitchenerb

\section{Article history}

Publication date: March 2015

Citation: Roberts N, Holden J, Duck T, Kitchener S. Health promotion 'on steroids': the value of an experiential approach to promote rapid HIV testing in NSW, Australia. Public Health Res Pract. 2015;25(2):e2521522. doi: http://dx.doi. org/10.17061/phrp2521522

\section{Key points}

- Making HIV testing easier and more accessible is critical in preventing transmission, through early detection and access to timely treatment and care

- A partnership approach was used to develop an innovative model of delivering and promoting community-based HIV testing

- Social media and experiential techniques have been used to engage target groups and the broader public in health promotion messages

\section{Abstract}

In 2012, there was a sharp increase in human immunodeficiency virus (HIV) notifications in New South Wales (NSW), Australia, following a 10-year period of relative stability. This increase, among other factors, triggered the development of a new HIV strategy that included renewed efforts to increase testing to improve early diagnosis, enable early treatment and reduce the risk of onward transmission. This article describes the activities conducted by NSW Health and partner organisations during November 2013 in the week before World AIDS Day (phase one (P1) ) and HIV testing week in July 2014 (phase 2 (P2)).

A model of pop-up HIV testing, new to Australia, was used to take testing to those most at risk of infection, and was promoted through social media and experiential techniques. During P1, an average of seven tests per hour were conducted at the pop-up service, compared with four tests per hour at a fasttrack screening service in a nearby sexual health clinic.

During HIV testing week, the campaign hashtag was mentioned an average of 56 times per day, following a baseline of six mentions per day one week before. The estimated total social media reach was 549769 people via 459 posts. The pop-up testing model proved popular, and the use of social media and experiential techniques has extended the reach of the 'test more' messages. Further research is required to determine causality between specific HIV messaging and experiential techniques and testing rates. 


\section{Background}

Human immunodeficiency virus (HIV) remains a public health concern with significant costs to the healthcare system. Men who have sex with men (MSM) are the population group most affected by HIV in Australia, with about two-thirds of new infections occurring in this group. ${ }^{1}$ Although HIV remains a chronic, incurable disease, approaches to HIV diagnosis, treatment and prevention have significantly evolved in recent years in response to emerging scientific evidence of the effectiveness of antiretroviral treatment as prevention and new rapid testing technologies. ${ }^{2}$ Regular HIV testing remains critical in preventing HIV transmission through early detection and access to timely treatment and care. ${ }^{3}$

\section{A new era}

New South Wales (NSW) has historically had the highest rate of HIV diagnoses in Australia, from the beginning of the epidemic until 2010. ${ }^{1}$ In 2012, following a 10-year period of relative stability, the number of HIV notifications in NSW sharply increased ${ }^{4}$, with 408 new notifications, a $24 \%$ increase compared with 2011. MSM accounted for $78 \%$ of all notifications. ${ }^{4}$

In addition, NSW released the NSW HIV strategy 2012-2015: a new era ${ }^{5}$ on World AIDS Day 2012. The strategy was more ambitious than previous strategies ${ }^{6}$, having the goals of virtually eliminating HIV transmission by 2020 , and reducing new infections by $60 \%$ among MSM by 2015, through increasing HIV testing and treatment, and safe sex practices. ${ }^{5}$

Successfully scaling up HIV testing to reduce the pool of individuals with undiagnosed HIV is a key component of HIV prevention and is critical to the successful implementation of the strategy. ${ }^{5}$ Interventions that increase testing, together with combination prevention measures and early treatment, are expected to correspond with net decreases in incident infections. ${ }^{2}$ This includes making testing easier and more accessible, disrupting traditional thinking about appropriate settings for HIV testing and increasing awareness among MSM about testing, particularly for those at high risk. ${ }^{5}$

Two implementation challenges identified by NSW Health included addressing resistance to change and parting with traditional approaches among sector stakeholders, and taking testing messages and services to target populations through unexplored service models that are innovative within Australia.

\section{Testing within the community}

HIV testing in NSW is traditionally provided in clinical settings such as general practice and public sexual health clinics. To achieve the new strategy targets, accessible service models, including rapid HIV testing $(\mathrm{RHT})$, were needed to achieve the high coverage and frequency of HIV testing required. The increasing availability of RHT in NSW has provided a quick and convenient model for HIV testing that is proving successful in attracting higher-risk MSM and identifying a significant number of new HIV infections (unpublished evaluation by ACON and Sydney Sexual Health Centre for NSW Ministry of Health). RHT allows clients to receive preliminary results within 30 minutes - more quickly than traditional testing methods. ${ }^{7}$ Although 18 public sexual health clinics incorporated $\mathrm{RHT}$, there was little innovation in exploring new RHT settings for people who did not access traditional models of testing. Community-based $\mathrm{RHT}$ is widespread in the US and Europe, and some community (clinic based) and outreach HIV testing has been identified in Australia, but there were no pop-up testing services as of $2013 .^{8,9}$ Increasing the convenience of $\mathrm{RHT}$, including in nonclinical settings, is likely to raise awareness of the importance of HIV testing and increase testing within groups at risk. ${ }^{7}$

We describe the activities held during the week before World AIDS Day 2013 - phase one (P1) - and HIV testing week in July 2014 - phase 2 (P2). To encourage services to offer HIV testing in more flexible ways and to raise awareness about rapid testing among MSM, Australia's first ever pop-up testing site was developed for P1. An alliance of key stakeholders and opinion leaders was formed to enable an integrated approach to the development, acceptability and operational aspects of the service. A NSW Health-branded caravan, staffed by one nurse and two peer educators ${ }^{9,10}$ (pop-up site) was installed in east Sydney for five days during P1. This location was chosen because of the concentration of MSM living and socialising in the area. During this period, 503 people engaged with the pop-up site, and 195 people requested a rapid HIV test (none of these people were diagnosed with a new HIV infection). Demand was high, averaging seven tests per hour, compared with four at a local clinic-based RHT service. ${ }^{9}$ Consequently, testing hours were extended. A study concluded that pop-up RHT was feasible and, in this situation, reached high-risk MSM. ${ }^{9}$

The 'test more' message was reinforced by a NSW Ministry of Health public relations and social media strategy to generate conversations about RHT in MSM, mainstream and health professional communities.

\section{Keeping testing top of mind}

A key process for health promotion practice is the communication of health messages and information. It involves the transfer of messages at both individual and community levels to educate and influence decisions to improve health. ${ }^{11}$ Strategies include, among others, social marketing (applying commercial marketing techniques to planning, executing and evaluating programs ${ }^{12}$ ), peer education and public relations. 
Experiential techniques, or actively involving people in a message ${ }^{13}$, were used in this initiative to enable the target audience to experience $\mathrm{RHT}$ firsthand. Compared with mass media campaigns, experiential events tend to communicate on a more personal level, and may generate a deeper level of emotional engagement through physical immersion in a message or brand. Although experiential learning has been used to train public health practitioners ${ }^{14}$, it is of interest that there is little literature relating to experiential approaches to communicating health promotion messages to the public.

The public health strategy used by NSW Health represented a facilitation of 'concrete experience'13, in which the pop-up service was used to both deliver the intervention and promote a public health message.

A welcoming environment was created at the pop-up caravan, and community-based peer workers put people at ease with being tested. The visual impact of the site operated at both the service user and community levels to promote the testing message (Figure 1).

Figure 1. Pop-up testing caravan in Taylor Square, Sydney

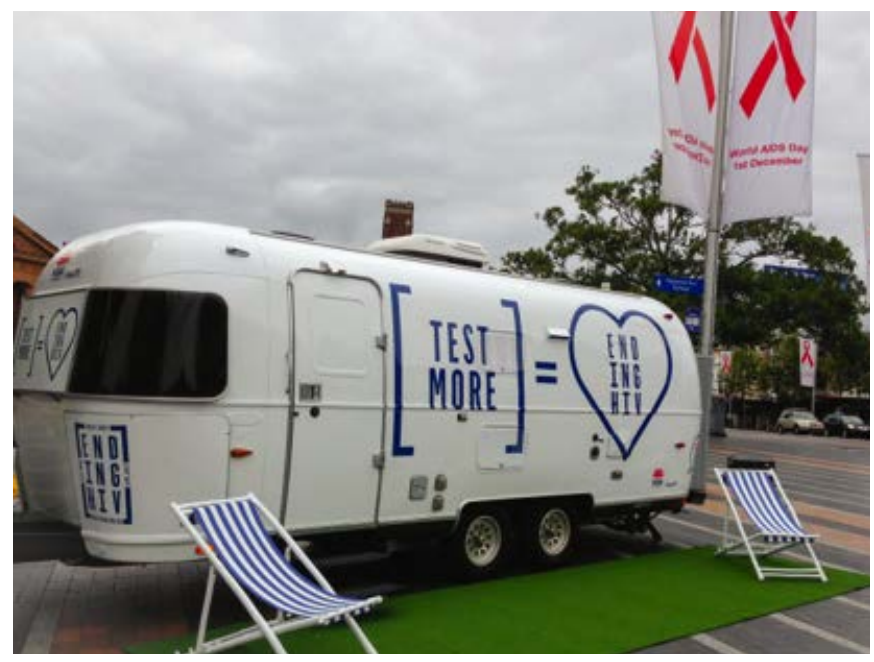

To build on the success of this approach, during P2 a similar pop-up testing bus was installed at the same location. The social media campaign used during P1 was amplified to include roving installations or 'hotspots' around inner Sydney aimed at encouraging the public to be photographed showing their support for the campaign, and to post their photos via social media using the hashtags \#HelpEndHIV and \#HIVTestingWeek. Brand ambassadors were used to further enable community ownership during the campaign and participation in the 'movement' to end HIV. Local businesses and community organisations also participated in social media activities to provide resonance for the community.

\section{Social media - targeting messages cost-effectively}

Social media, including Facebook, YouTube and Twitter, are increasingly being used by the public for healthrelated issues ${ }^{15}$ and have been recognised by the World Health Organization as being particularly useful in public health for facilitating direct communication with the public. ${ }^{16}$ Evidence suggests that multimedia campaigns in developed countries can significantly increase HIV testing among MSM, but that further, more rigorous, research is needed. ${ }^{17}$

In 2012-13, 81\% of households in NSW had internet access $^{18}$, suggesting that internet-based applications are essential mechanisms for the promotion of public health messages. Further, internet usage via smart phones grew from 13\% of the population in 2010 to $42 \%$ in 2013, suggesting that people now have increased opportunity to share public health messages when they experience them. ${ }^{19}$

Social media mentions of the hashtags \#HelpEndHIV and \#HIVTestingWeek were analysed for P2, as well as for the preceding and following week. Peaks in the number of mentions of these hashtags corresponded to activities taking place as part of the social media campaign. During P2, the average number of daily mentions was 56, following a baseline of 6 mentions per day one week earlier.

By analysing the number of people who shared the campaign hashtags, combined with follower numbers, it was possible to estimate the total reach of overall social media dialogue during the three-week period. The estimated total reach was 549769 people via 459 individual posts (unpublished Buzz Numbers report to NSW Health). However, this is likely to be an underestimate, because of privacy settings on some social media platforms, which occasionally restrict monitoring of reach.

\section{Media engagement}

It was essential to engage media (community and mainstream) to facilitate targeted and universal approaches to communicating the health promotion message to test more. Media engagement for the period around P1 and P2 was analysed. During P1, more than 30 mainstream, gay and online media channels published stories about the campaign, including in health and medical publications, with an estimated audience of more than four million people. For P2, there were 53 published stories, with an estimated audience of more than one million people (unpublished report by MediaPortal to NSW Government). 


\section{Addressing concerns}

The campaign aimed to allay any concerns about the viability, acceptability and rigour of pop-up HIV testing among individuals, clinicians and the broader heath sector. This was addressed by communicating the message across multiple platforms, including traditional and social media as well as industry publications.

Structures and mechanisms set in place by NSW Health to coordinate activities across multiple stakeholders (including the alliance of key stakeholders and opinion leaders) worked to facilitate a common understanding. They also helped to further momentum towards embedding contemporary messaging and service delivery within the health sector.

\section{Conclusion and implications}

Recent increases in testing have been evident at the state level. In publicly funded sexual health clinics in NSW, where longer-term trend data are available, there were consistent increases in the number of HIV tests conducted with MSM during the first six months of 2014 (an increase of 29\% compared with the average number of tests per quarter in 2013). ${ }^{4}$ Levels of testing vary across NSW, but data suggest that there has been ongoing success in efforts to increase testing, particularly among high-risk groups. ${ }^{4}$ The demonstrated uptake of pop-up testing by people at high risk has since provided momentum for both Northern NSW and Mid North Coast Local Health Districts to establish two pop-up RHT services. This suggests initial engagement of the broader NSW health system with the potential for pop-up RHT.

The use of social media and experiential techniques has extended the reach of the 'test more' messages considerably. However, further research is required to determine the level of causality between such techniques and testing rates, particularly in the context of other simultaneous health promotion activities relating to HIV. Further evaluation of the communication of public health messages by NSW Health and the frequency of testing and retesting among groups at high risk is required to ensure ongoing effectiveness.

Leadership from all levels of the NSW Government and support from community groups, peak bodies and other stakeholders provided mobilisation and commitment towards promoting testing in 2014. It is important to use a mix of testing settings (including pop-up RHT) to further increase testing, particularly among MSM, to identify HIVpositive individuals and link them to care, and to reduce the pool of undiagnosed people.

\section{Acknowledgements}

This work was completed while NR was a trainee on the NSW Public Health Training Program, funded by the NSW Ministry of Health. He undertook this work while based in the Centre for Population Health, NSW Ministry of Health. The NSW Ministry of Health extends thanks to its partner organisations on this project: ACON, St Vincent's HIV Reference Laboratory, South Eastern Sydney Local Health District and the Kirkton Road Centre.

\section{Competing interests}

NR was employed on a casual basis as a consultant evaluator by ACON between July 2013 and December 2013, before this manuscript was written. Although ACON is referred to in this manuscript, the program subject to that evaluation ('Mind and matter') is separate to the ACON initiatives referred to here.

\section{References}

1. The Kirby Institute. HIV, viral hepatitis and sexually transmissible infections in Australia: annual surveillance report 2013. Sydney: The Kirby Institute, University of New South Wales; 2013.

2. Jansson J, Kerr C, Wilson D. Predicting the population impact of increased HIV testing and treatment in Australia. Sex Health. 2014;11(2):146-54.

3. Attia S, Egger M, Muller M, Zwahlen M, Low N. Sexual transmisison of HIV according to viral load and antiretroviral therapy: systematic review and metaanalysis. AIDS. 2009;23(11):1397-404.

4. Health Protection NSW, NSW Health. NSW HIV/ AIDS database. Sydney: NSW Health; 2014 [cited 2014 Oct 20]; [about 2 screens]. Available from: www.healthstats.nsw.gov.au/Indicator/com_hivaidsnot

5. NSW Health. NSW HIV Strategy 2012-2015: a new era. Sydney: NSW Ministry of Health; 2012 [cited 2014 Oct 20]. Available from: www.health.nsw.gov.au/ publications/Publications/nsw-hiv-strategy-2012-15.pdf

6. NSW Health. NSW HIV/AIDS Strategy: 2006-2009. Sydney: NSW Health; 2012.

7. Gray R, Prestage G, Down I, Ghaus M, Hoare A, Bradley J, et al. Increased HIV testing will modestly reduce HIV incidence among gay men in NSW and would be acceptable if HIV testing becomes convenient. PLoS One. 2013;8(2):e55449.

8. Pedrana A, Guy R, Bowring A, Hellard M, Stoove M. Community models of HIV testing for men who have sex with men (MSM): systematic review 2011. Commissioned by ACON. Melbourne: Burnet Institute; 2011.

9. Knight V, Gale M, Guy R, Parkhill N, Holden J, Leeman C, et al. A novel time-limited pop-up HIV testing service for gay men in Sydney, Australia, attracts high-risk men. Sex Health. 2014;11(4):345-50. 
10. Gale M, Holden J, Selvey C, Chant K, Whittaker B. Eliminating HIV transmission in New South Wales: the critical role of testing. Med J Aust. 2014;201(5):260-2.

11. Centers for Disease Control and Prevention: CDC 24/7 saving lives, protecting people. Atlanta, GA: Centers for Disease Control and Prevention. Division of nutrition, physical activity, and obesity: social marketing resources; 2011 Aug 8 . [cited 2014 Oct 20]; [about 3 screens]. Available from: www.cdc.gov/nccdphp/DNPAO/ socialmarketing/

12. Wei $C$, Herrick A, Raymond $H$, Anglemyer A, Gerbasse A, Noar S. Social marketing interventions to increase HIV/STI testing uptake among men who have sex with men and male-to-female transgender women. Cochrane Database Syst Rev. 2011;(9):CD009337.

13. Kolb D. Experiential learning. New Jersey: Prentice Hall; 1984.

14. Cornelius J. To be touched by AIDS: an HIV-experiential teaching method. J Nurs Educ. 2004;43(12):576.

15. Korda $\mathrm{H}$, Itani Z. Harnessing social media for health promotion and behaviour change. Health Promot Prac. 2013;14(1):15-23.
16. McNab C. What social media offers to health professionals and citizens. Bull World Health Organ. 2009;87(8):566.

17. Lewis M, Uhrig J, Ayala G, Stryker J. Reaching men who have sex with men for HIV prevention messaging with new media: recommendations from an expert consultation. Annals of the Forum on Collaborative HIV Research; 2011;13(3):11-18.

18. Australian Bureau of Statistics. Household use of information technology, Australia, 2012-13. Canberra: Australian Bureau of Statistics; 2014 [cited 2014 Oct 20] Available from: www.abs.gov.au/AUSSTATS/abs@.nsf/ DetailsPage/8146.02012-13?OpenDocument

19. Australian Communications and Media Authority. Communications report 2012-2013. Sydney: Australian Communications and Media Authority; 2014 [cited 2014 Oct 20]. Available from: www.acma.gov.au/ theACMA/Library/Corporate-library/Corporatepublications/communications-report-2012-13

\section{Copyright: (c)(3) (-)}

(C) 2015 Roberts et al. This article is licensed under the Creative Commons Attribution-NonCommercial-ShareAlike 4.0 International Licence, which allows others to redistribute, adapt and share this work non-commercially provided they attribute the work and any adapted version of it is distributed under the same Creative Commons licence terms. See: www.creativecommons.org/licenses/by-nc-sa/4.0/ 